\title{
8 \\ A Fast Routing and Bandwidth Management Method in ATM Networks
}

\author{
Mitsuaki Kakemizu, Masahiro Taka \\ Ultra-high Speed Network and Computer Technology Laboratories \\ 2-6 Toranomon 5-Chome, Minato-ku Tokyo 105, Japan \\ Telephone: +81-3-3578-9361 Fax:+81-3-3578-8184 \\ E-mail:kake@magical.egg.or.jp
}

\begin{abstract}
Current supercomputers and workstations operate at 100 GFLOPS-TFLOPS and at 10-100 MFLOPS, respectively, and these processor speeds will increase in the future. This has led to an increasing demand for the real-time transfer of scientific high-dimensional image data, and ATM networks would therefore have to provide user-network interfaces with bandwidths in the gigabit range from now on. Because computer communication usually produces unpredictable burst traffic, it is difficult for the bandwidth reservation method based on statistical multiplexing to use bandwidths efficiently. If the connectionless server approach were adopted, these servers would cause throughput bottlenecks in gigabit applications. As a result, this paper proposes a fast routing and bandwidth management method that can effectively transport gigabit traffic. It also undertakes performance evaluation by comparing the proposed method with the P-NNI method that the ATM Forum proposes.
\end{abstract}

\section{Keywords}

Routing, bandwidth management, virutual channel connection, hierarchy, ATM

\section{BACKGROUND}

The Asynchronous Transfer Mode (ITU-T, 1990) is expected to be the basic technology providing multimedia services in a B-ISDN. Its features include bit-rate flexibility and statistical multiplexing capability, and ATM networks are also expected to be used in transferring scientific image data generated by supercomputers. Such an application is characterized by high-speed burst traffic, however, and when the amount of burst traffic exceeds the traffic-handling capability of a network using statistical multiplexing, degradation in service quality such as cell loss occurs. To prevent network congestion, statistical multiplexing requires that each traffic source notifies all QOS parameters such as peak cell rate, average cell rate, and cell loss ratio in the call set-up phase. Only a few computer communication applications, however, are able to accurately specify average cell rate in the call set-up phase. This is because the average cell rate changes dynamically and cannot be 
predicted. As a result, it may not be appropriate to introduce statistical multiplexing for handling such high-speed burst traffic in ATM networks.

ITU Recommendation I.364(ITU-T, 1992) proposes that ATM networks make use of connectionless servers (CLSs) to support connectionless services in the megabit range. These servers would be located at specific ATM nodes, and the source data terminal equipment (DTE) accommodated in one of the nodes would send cells to a CLS by default routing. The CLS would then route the cells to the other CLS at the destination ATM node. Consequently, the CLS would cause a bottleneck in gigabit applications because the ATM connection must always be terminated at each CLS and a Connectionless Network Access Protocol or Connectionless Network Interface Protocol must be processed for the first cell belonging to the protocol data unit of the ATM adaptation layer (AAL)-3/4 in each CLS.

\section{BASIC CONCEPT}

One of the most effective methods is FRP (Fast Reservation Protocol) (Boyer, 1992), which selects a route in the call set-up phase and reserves bandwidths along the route on a burst-by-burst basis in the call. This method, however, would increase burst blocking probability especially in gigabit applications and would result in long connection delay for back-off timer delay because network states during each burst reservation phase are different from states in the call set-up phase due to the dynamic behavior of available bandwidth resources in networks.

Another effective method is the P-NNI(Private NNI) Draft Specification(ATM Forum, 1995), which calculates the optimal route on a burst-by-burst basis and reserves bandwidth on the optimal route on an in-series and link-by-link basis. However, this method would also result in long connection delay because it requires the optimal route to be calculated on a burst-by-burst basis.

We therefore used an approach that allows the optimal route to be rapidly selected and reserves bandwidth along the route on a burst-by-burst basis. We propose a routing and bandwidth management method (Kakemizu, 1995) that efficiently supports gigabit computer communications applications such as the real-time transfer of scientific high-dimensional image data and medical image data. The main features of this method are as follows:

1 Separation between the VCC establishment procedure and the management of routing tables.

As this method periodically calculates the optimal route between necessary nodes without being synchronized with the VCC establishment procedure, route selection can be performed rapidly when establishing a VCC because the only procedure required is to refer to the predetermined route.

1 Division of ATM networks into several subnetworks and the concept of hierarchical subnetworks.

This method not only allows ATM networks to be divided into several subnetworks, but also allows larger subnetworks to be divided into smaller subnetworks if necessary. VCC establishment processing is performed based on the scope of each subnetwork independent of other subnetworks, and the management of routing tables is also performed independent of all lower subnetworks and other subnetworks in different upper subnetworks. The P-NNI method also adopts the division and hierarchy of networks. However, the extent of routing table in each subnetwork using this method is smaller than that in each ATM node in each subnetwork called the peer group in the P-NNI method. This is because the P-NNI method requires each ATM node in networks to manage not only the routing table in the lowest peer group that it belongs to but also the routing tables in all upper peer groups that it belongs to. This is even though all the peer groups and links in all upper peer groups that it belongs to are logically abstracted. This 
distributed processing in the proposed method would therefore make it more suitable for largescale networks than the P-NNI method.

Parallel execution of route selection and bandwidth reservation procedures in each lower subnetwork in compliance with request from the common upper subnetwork.

As shown in figure 1, although each VCC establishment is performed in-series from upper subnetworks toward lower subnetworks between the source and destination DTE, route selection and bandwidth reservation procedures for VCC establishment in all subnetworks in a common upper subnetwork are executed in parallel in compliance with requests from the common upper subnetwork. As a result, this method allows the establishment period of all VCs in each subnetwork to be almost constant independent of the sum of subnetworks/nodes in the subnetwork while conventional methods (including P-NNI method and FRP method) result in a VCC establishment time that is proportional to the sum of the nodes between the source DTE and destination DTE. This is because these conventional methods execute processing on an inseries and link-by-link basis.

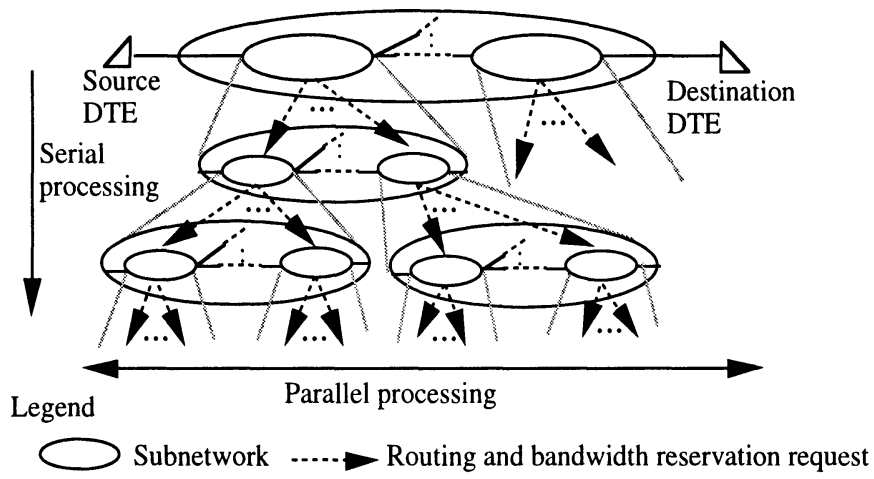

Figure 1 Conceptual procedure for VCC establishment

Simplification of signalling procedures by sending RM-cells through dedicated PVCs. Permanent Virtual Channels (PVCs) have been established in advance between all DTEs that may establish VCCs based on the proposed method and the subscriber node accommodating these DTEs, and between all adjacent nodes. Because these PVCs are only used to transfer resource management cells (RM-cells) for VCCs establishment based on this method, the proposed method can prevent the protocol overheads of AAL-5 and signalling protocols such as Q.2931(ITU-T, 1993), UNI Signalling 4.0(ATM Forum, 1995) and Q.2764(ITU-T, 1995). This method can also simplify signalling procedures.

\section{RAPID METHOD FOR ESTABLISHING VIRTUAL CHANNEL CONNECTIONS}

\subsection{Network model}

Figure 2 shows the network model on which our proposed method is based. Networks can be divided into several virtual subnetworks, and each subnetwork can be divided into several smaller virtual 
subnetworks. Thus, there are hierarchical relationships between subnetworks, and the network model is recursive.

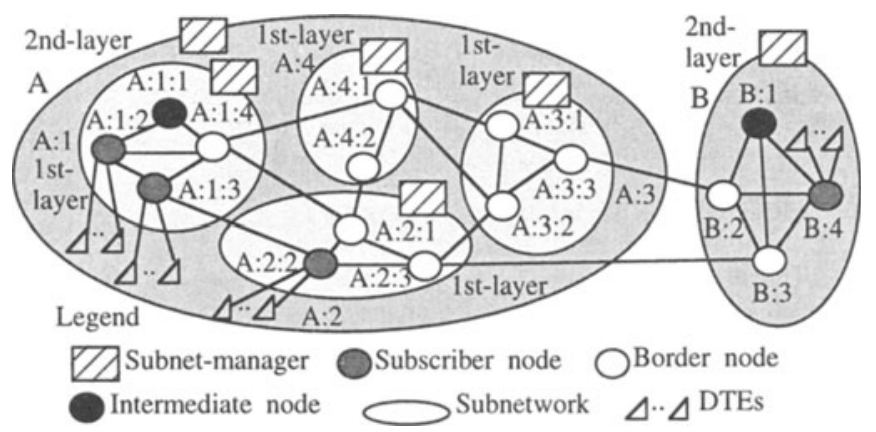

Figure 2 Network model.

One subnet-manager, which is responsible for routing and requesting VCC establishment, is assigned in each subnetwork. Each subnet-manager may be implemented in any physical node in the subnetwork that the subnet-manager belongs to or may be implemented in a dedicated node independent of other physical nodes in the subnetwork. PVCs for carrying routing information and signalling messages are established in advance between each node and all subnet-managers receive routing information from this node, between upper and lower subnet-managers, and between each subscriber node and all subnet-managers accommodating it. PVCs for exchanging routing information are also established in advance between subnet-managers that belong to subnetworks in the common upper subnetwork. VCC establishment processing is performed by each subnet-manager independent of the subnet-managers in other subnetworks, and the management of routing tables is also performed by each subnet-manager independent of the subnet-managers in other subnetworks in the different upper subnetwork. As noted in Section 2, such distributed processing makes this method suitable for large-scale networks.

\subsection{Management of routing tables}

Routing tables consist of an inter-subnetwork routing table and an intra-subnetwork routing table. The former is managed by all subnet-managers unless there is only one subnetwork in the network, and the latter is managed only by subnet-managers that belong to the lowest subnetworks. The intersubnetwork routing table shown in Table 1-(a) is used to select an optimal route between any two subnetworks extracted from all subscriber subnetworks and all border subnetworks in the common upper subnetwork. The subscriber subnetworks shown as 'A', 'A:1', 'A:2' or 'B' in Figure 2 are subnetworks accommodating at least one subscriber node that itself accommodates at least one DTE. The border subnetwork shown as 'A:3' in Figure 2 is a subnetwork connected to other subnetworks contained in a different upper subnetwork and is not a subscriber subnetwork. The intra-subnetwork routing table shown in Table 1-(b) is used to select an optimal route between any two nodes extracted from all subscriber nodes and all border nodes in the common lowest subnetwork. The border node shown in Figure 2 stands for a node connected to other nodes contained in a different lowest subnetwork and is not a subscriber node. The optimal physical link in Table 1 is a link with the maximum available bandwidth of all physical links between subnetworks. Each bottleneck bandwidth 
in Table 1 is the minimum available bandwidth for all links on the route between the source subnetwork and the destination subnetwork or between the source node and the destination node. The optimal route shown in this paper is defined as the route with the least bottleneck bandwidth consisting of the fewest hops.

Table 1 Examples of routing tables

(a) Inter-routing table for each subnetwork A:1-4 in subnetwork A in Figure 2.

\begin{tabular}{lllll}
$\begin{array}{l}\text { Source/Destination } \\
\text { subnetwork id }\end{array}$ & $\begin{array}{l}\text { Number of } \\
\text { hops on route }\end{array}$ & $\begin{array}{l}\text { Bottleneck } \\
\text { bandwidth (Gbps) }\end{array}$ & $\begin{array}{l}\text { Subnetwork } \\
\text { id on route }\end{array}$ & Optimal phisical link \\
\hline $1-2$ & 1 & 2.5 & $1-2$ & $1: 3-2: 2$ \\
& 2 & 2 & $1-4-2$ & $1: 4-4: 1,4: 2-2: 1$ \\
& 3 & 3 & $1-4-3-2$ & $1: 4-4: 1,4: 1-3: 2,3: 2-2: 3$ \\
$1-3$ & $\ldots$ & $\ldots$ & $\ldots$ & $\ldots$ \\
$2-3$ & $\ldots$ & $\ldots$ & $\ldots$ & $\ldots$ \\
\hline
\end{tabular}

(b) Intra-routing table for subnetwork A:1 in Figure 2.

\begin{tabular}{llll}
\hline $\begin{array}{l}\text { Source/Destination } \\
\text { node id }\end{array}$ & $\begin{array}{l}\text { Number of } \\
\text { hops on route }\end{array}$ & $\begin{array}{l}\text { Bottleneck } \\
\text { bandwidth (Gbps) }\end{array}$ & $\begin{array}{l}\text { Node id } \\
\text { on route }\end{array}$ \\
$2-3$ & 1 & 1.5 & \\
& 2 & 2 & $2-4-3$ \\
$2-4$ & 3 & 2.3 & $2-1-4-3$ \\
$3-4$ & $\ldots$ & $\ldots$ & $\ldots$ \\
\hline
\end{tabular}

Each subnet-manager is periodically notified of the available bandwidth for all physical links between the subnetwork that it belongs to and other subnetworks directly connected to it from all nodes that manage their links. Each subnet-manager also periodically exchanges this information with other subnet-managers in the common upper subnetwork. After this, each subnet-manager calculates the optimal route by arranging all routes between both source/destination subnetworks as mentioned above, in ordering fewer hops and a broader bandwidth to create less bottlenecking. Similarly, after each individual subnet-manager belonging to the lowest subnetwork has received information on the available bandwidth for each physical link between all pairs of nodes in the subnetwork from all nodes that manage their links, it calculates the optimal route by arranging all routes between both source/destination nodes as mentioned above, in ordering fewer hops and a broader bandwidth to create less bottlenecking. Because these arrangements are executed periodically, without being synchronized with the VCC establishment procedure, route selection when establishing a VCC can be performed rapidly as the only procedure required is a reference to the most desirable predetermined route of the routes arranged last. There is also another option: that each node does not send the available bandwidth periodically but only when the difference between it and the last available bandwidth sent exceeds a certain threshold.

\subsection{VCC establishment procedures}

As shown in Figure 3, a VCC is established as follows:

1. The source node:

a) receives VCC_req and reserves the link bandwidth between the source node and source DTE.

b) sends VCC_req_ack to the source DTE.

c) analyzes both the network prefix of the source DTE address and the network prefix of the destination DTE address, and sends VCC_req to one of the $n$ th-layer SMs called the $n$ th-layer 
responsible SM. Address format in the proposed method is identical to the one in the ATM Forum (ATM Forum, 1995), and includes a network prefix that represents the configuration for each DTE or node or subnetwork in a hierarchical structure for networks, and this network prefix assists the subnet-manager to select the optimal route on a burst-by-burst basis. This SM is assigned by the subnetwork accommodating the source node in the lowermost common layer where both the subnetwork accommodating source node and the subnetwork accommodating destination node coexist.

2. The $n$ th-layer responsible SM:

a) receives VCC_req and selects the optimal route between the subnetwork that the $n$ th-layer responsible $\overline{\mathrm{SM}}$ belongs to and the other subnetwork that the message transmitting SM shown in Figure 3 belongs to by referring to the inter-routing table.

b) sends VCC_req to all nodes as shown in Figure 3 and the destination node through the message transmitting SM on the optimal route. Then, each of these nodes in-parallel independently reserves bandwidth on the link designated by the VCC_req.

c) sends Conn_Ack to all these nodes except the destination node and sends VCC_req to some ( $n$ 1)th-layer SMs, termed ( $n-1)$ th-layer responsible SMs, after receiving Conn from all nodes. Each of these $(n-1)$ th-layer responsible SMs is separately assigned by the $(n-1)$ th-layer subnetwork in each $n$ th-layer subnetwork on the optimal route. Then, each of these nodes except the destination node in-parallel independently establishes the virtual channel.

d) sends Conn_Ack to all the $(n-1)$ th-layer responsible SMs after receiving Conn from them.

3. Until each lowest-layer SM receives VCC_req from the same layer SM, each (n-1)th-layer responsible SM in-parallel and recursively:

a) receives VCC_req and selects the optimal route between the subnetworks designated by the VCC_req by referring to the inter-routing table.

b) sends VCC_req to all the nodes as shown in Figure 3 on the optimal route. Then, each of these nodes in-parallel independently reserves bandwidth on the link designated by the VCC_req.

c) sends Conn_Ack to all these nodes and sends VCC_req to $(n-2)$ th layer SMs that are $(n-2)$ thlayer responsible SMs, after receiving Conn from all nodes. Each of these $(n-2)$ th-layer responsible SMs is separately assigned by the $(n-2)$ th-layer subnetwork in each $(n-1)$ th-layer subnetwork on the optimal route. Then, each of these nodes in-parallel independently establishes the virtual channel. If there is no $(n-2)$ th-layer SM, the $(n-1)$ th-layer SM sends VCC_req to all same-layer SMs on the optimal route.

d) sends Conn_Ack to all the ( $n$-2)th-layer responsible SMs after receiving Conn from them.

e) sends Conn to the $n$ th-layer responsible SM.

4. After each lowest-layer SM receives VCC_req from the same-layer SM, each lowest-layer SM, in-parallel:

a) selects the optimal route between the nodes designated by the VCC_req by referring to the intrarouting table.

b) executes the same procedure as shown in 3.b).

c) sends Conn_Ack to all these nodes after receiving Conn from these nodes, and then each of these nodes in-parallel independently establishes the virtual channel.

d) sends Conn to the SM having received VCC_req.

5. The $n$ th-layer responsible SM sends Conn to the source DTE through the source node and sends Conn_Ack to the destination node through the message transmitting SM.

6. The source node receives Conn_Ack from the source DTE and establishes the virtual channel.

7. The destination node establishes the virtual channel and sends Conn_Ack to the destination DTE. 


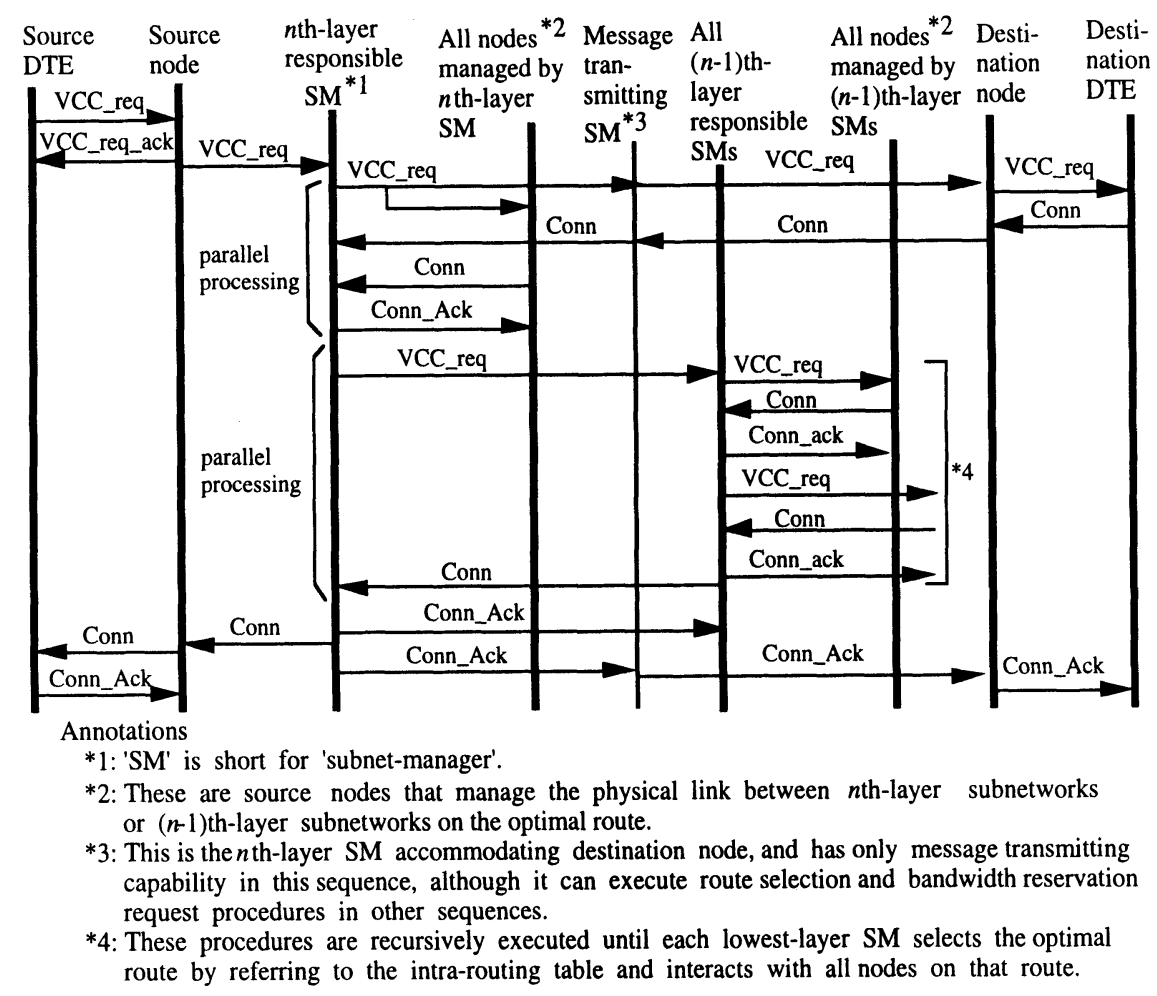

Figure 3 Sequence chart for VCC establishment.

As mentioned above, the proposed method allows VCCs to be established more rapidly than conventional methods because route selection and bandwidth reservation procedures for VCC establishment are executed in-parallel in all subnetworks and all nodes between source DTE and destination DTE. In contrast, conventional methods have procedures on a link-by-link basis and inseries.

\subsection{Comparison with other methods}

Because the FRP method must always reserve bandwidth on the only route selected in the call set-up phase on a burst-by-burst basis, this method tends to increase burst blocking probability especially in gigabit applications. Moreover, this method reserves bandwidth on a link-by-link basis and in-series. As a result, this method would result in long connection delays. On the other hand, the P-NNI method reserves bandwidths on the optimal route on a burst-by-burst basis and decreases burst-blocking probability like the proposed method. However, the P-NNI method takes more time in establishing VCCs than the proposed method because it needs to calculate the optimal route just after VCC establishment request from the source DTE or the source peer group. It also needs to reserve bandwidth on an in-series and link-by-link basis while the VCC the establishment procedure for the proposed method refers to the optimal route calculated just before VCC establishment request from the source node or upper SM, in-parallel to reserve bandwidth on the route. 


\section{PERFORMANCE EVALUATION}

\subsection{Simulation model}

Figure 4 shows the traffic model that was used to evaluate the proposed method by simulation. Burst duration denotes the duration for any terminal to send burst data consisting of many ATM cells to the subscriber node accommodating the terminal, and idle duration denotes the duration for any terminal not sending any ATM cells to the subscriber node. The necessary bandwidth denotes the bandwidth to send burst data for the burst duration, and is assumed to be exponentially distributed on a burst-byburst basis and to be constant for each burst duration. Burst duration and idle duration are also assumed to be exponentially distributed with average length of $100[\mathrm{~ms}]$ and $900[\mathrm{~ms}]$ on a burst-byburst basis, respectively. Inter-burst arrival and burst duration distributions for each terminal are independent. The number of reattempts for each blocked burst is equal to zero. The aggregate arrival process is assumed to be a Poisson distribution.

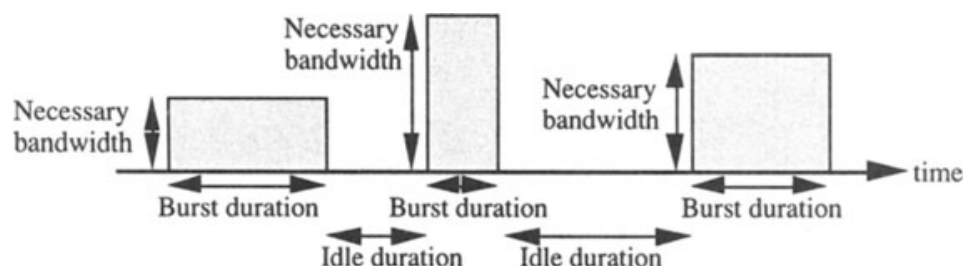

Figure 4 Traffic model.

Figure 5 shows the network topology used to evaluate the proposed method by simulation. Each Source 1-5 and each Destination 1-5 consists of 16 terminals independent of one another, respectively. Each terminal in each Source 1-5 establishes and releases VCC on a burst-by-burst basis to send each terminal in each Destination 1-5 burst data based on the burst generation style in Figure 4 through the network in Figure 5. Propagation delay between the subscriber nodes Switch 1 or Switch 8 and each terminal in each Source 1-5 or Destination 1-5 is assumed to be zero, and each propagation delay between nodes or between the subnet-manager and each node is assumed to be a value in proportion to a distance of $2[\mathrm{~km}]$. Each link bandwidth between the subnet-manager and all ATM nodes is 1 [Mbps] and each link bandwidth between ATM nodes and each link bandwidth between the subscriber nodes and 16 terminals in each Source 1-5 or Destination 1-5 are respectively shown in Figure 5.

The proposed method has only been compared with the P-NNI method because of the increased connection time as a result of high burst blocking probability with FRP. Performance of the suggested method is evaluated in the next section through use of a simulator that includes not only the traffic model and network topology in Figures 4-5 but also the functions of both the proposed and P-NNI methods. 


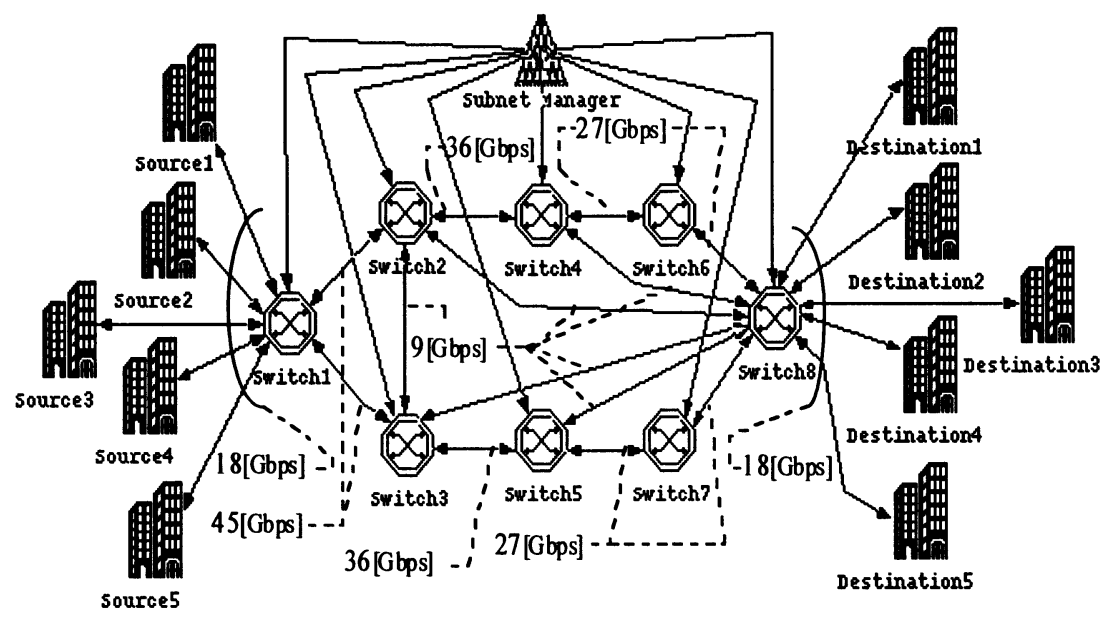

Figure 5 Network topology.

\subsection{Consideration}

Figure 6 shows the execution results for the simulator mentioned in the previous section and compares VCC establishment time between the proposed and P-NNI methods. The transverse axis shows the average necessary bandwidth per burst requested to be sent by each terminal in Source 1-5. That is, the average necessary bandwidth $B_{a v}$ can be defined by the following

$$
B_{a v}=\frac{1}{n} \sum_{i=1}^{n}\left(\frac{1}{k} \sum_{j=1}^{k} B_{i j}\right)
$$

where $n(=5 * 16)$ is the number of source terminals, $k$ is the sum of burst data that each source terminal requests the network to send to the corresponding destination terminal, and $B_{i j}$ is the necessary bandwidth of the $j$ th-burst data requested (including failure to send) by the $i$ th-source terminal. The vertical axis shows the average VCC establishment time for all burst data, that is, the average VCC establishment time $T_{a v}$ can be defined by the following

$$
T_{a v}=\frac{1}{\mathrm{n}} \sum_{i=1}^{n}\left(\frac{1}{m} \sum_{j=1}^{m} T_{i j}\right)
$$

where $m$ is the total number of burst data intervals successfully sent by each source terminal to the corresponding destination terminal, and $T_{i j}$ is the VCC establishment time of the $j$ th-burst data successfully sent by the $i$ th-source terminal. Figure 6 shows that reference to the predetermined route, simplification of signalling procedures, and parallel execution of route selection and bandwidth reservation procedures shorten the average VCC establishment time in the proposed method in comparison to the P-NNI method. In particular, the average VCC establishment time for the P-NNI 
method increases with increasing average necessary bandwidth, while it is almost constant when using the proposed method. This therefore proves the effectiveness of parallel execution of route selection and bandwidth reservation procedures because routes with more hops are often selected in establishing VCCs due to the lack of bandwidths on routes with fewer hops.

O Proposed method PCC establishent tine

\section{$\diamond \mathrm{P}$ - HirI nethod}

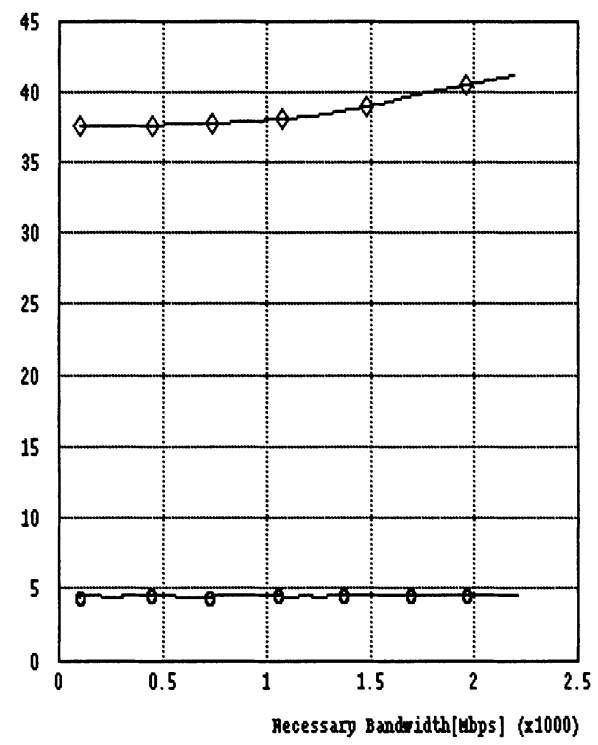

O Proposed method surst blocking probability

$\Delta \mathrm{P}$ - Rir wethod

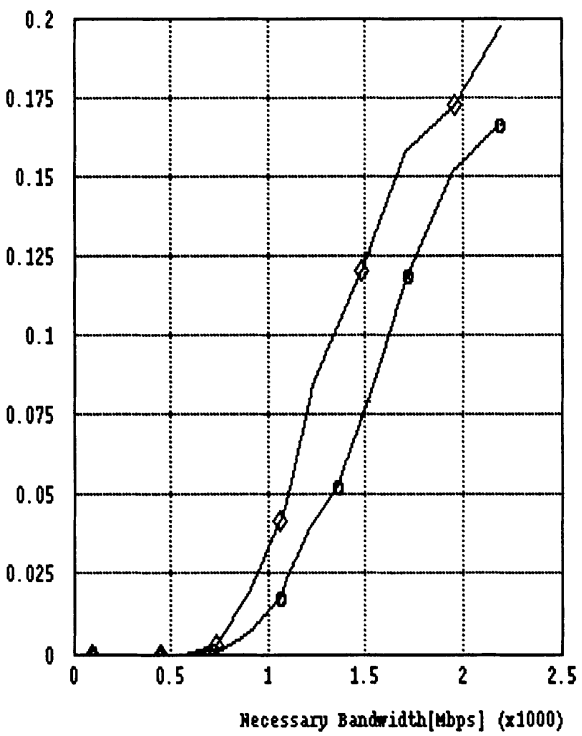

Figure 6 Connection time comparison.

Figure 7 Burst blocking probability comparison.

Figure 7 shows the results of execution for the simulator mentioned in the previous section and compares burst blocking probability between the proposed method and the P-NNI method. The transverse axis is identical to the one in Figure 6. The vertical axis shows average burst blocking probability for all burst data. That is, average burst blocking probability $P_{a v}$ can be defined by the following

$$
P_{a v}=\sum_{i=1}^{\mathrm{n}} F_{i} / \sum_{i=1}^{n} R_{i}
$$

where $R_{i}$ is the sum of burst data requested to be sent by the $i$ th-source terminal, and $F_{i}$ is the sum of burst data that the $i$ th-source terminal fails to send to the corresponding destination terminal. Figure 7 shows that the proposed method can more efficiently use the bandwidth resource in the network shown in Figure 5 than the P-NNI method. This is not only because VCC establishment time is shortened, but also because VCC release time is shortened by the parallel execution of bandwidth release procedures and the simplification of signalling procedures. As a result, the proposed method can rapidly utilize bandwidth resources. 


\section{CONCLUSIONS}

This paper has proposed a fast routing and bandwidth management method for ATM networks. The proposed method has four features: separation of the VCC establishment procedure from the management of routing tables, division of ATM networks into several hierarchical subnetworks, parallel execution of route selection and bandwidth reservation procedures in each subnetwork, and simplification of signalling procedures by sending RM-cells through dedicated PVCs. Performance evaluation showed that the proposed method enables each VCC to be established faster than with the P-NNI method. It can be deduced that the proposed method also enables each VCC to be established faster than by the FRP method. This is because the FRP method causes higher burst blocking probability especially in gigabit applications than the proposed method and reserves bandwidth on an in-series and link-by-link basis. Performance evaluation also showed that the proposed method is able to make more efficient use of the bandwidth resource than the P-NNI method.

\section{REFERENCES}

ATM Forum (1995) UNI Signalling 4.0, ATM Forum/94-1018R7.

ATM Forum (1995) P-NNI Draft Specification, ATM Forum/94-0471R13.

Boyer P.E. et al. (1992) A reservation principle with applications to the ATM traffic control, Computer Networks and ISDN Systems, Vol. 24.

ITU-T (1990) Broadband Aspects of ISDN, Recommendation I.121 (Draft).

ITU-T (1992) Support of Broadband Connectionless Data Service on B-ISDN, Recommendation I.364 (Draft).

ITU-T (1993) B-ISDN DSS2 Signalling for Basic Call/Connection Control, Recommendation Q.2931 (Draft).

ITU-T (1995) B-ISDN, B-ISDN User Part - Basic Call Procedures, Recommendation Q.2764 (Draft).

Kakemizu M. et al. (1995) A Fast Method for Establishing Virtual Channel Connections in Gigabit ATM Networks, Asia Pacific Conference on Communications '95, pp. 780-784. 